\title{
Novel one-step synthesis of silica nanoparticles from sugarbeet bagasse by laser ablation and their effects on the growth of freshwater algae culture
}

\author{
Nalan Oya San ${ }^{a, b, c}$, Canan Kurşungöz ${ }^{c}$, Yasin Tümtaş ${ }^{c}$, Öncay Yaşa ${ }^{c}$, Bülend Ortaç ${ }^{c} *$, \\ Turgay Tekinay ${ }^{\mathrm{a}, \mathrm{b}, * *}$ \\ a Polath Science and Literature Faculty, Biology Department, Gazi University, Ankara 06900, Turkey \\ ${ }^{\mathrm{b}}$ Life Sciences Application and Research Center, Gazi University, Ankara 06830, Turkey \\ ' UNAM Institute of Materials Science and Nanotechnology, Bilkent University, Ankara 06800, Turkey
}

\section{A R T I C L E I N F O}

\section{Article history:}

Received 21 September 2013

Received in revised form 6 November 2013

Accepted 25 November 2013

\section{Keywords:}

Laser ablation

One-step synthesis

Raman

Silica nanoparticle

Microalgae

\begin{abstract}
A B S T R A C T
Scientific research involving nanotechnology has grown exponentially and has led to the development of engineered nanoparticles (NPs). Silica NPs have been used in numerous scientific and technological applications over the past decade, necessitating the development of efficient methods for their synthesis. Recent studies have explored the potential of laser ablation as a convenient way to prepare metal and oxide NPs. Due to its high silica content, low cost, and widespread availability, sugarbeet bagasse is highly suitable as a raw material for producing silica NPs via laser ablation. In this study, two different NP production methods were investigated: laser ablation and $\mathrm{NaOH}$ treatment. We developed a novel, one-step method to produce silica NPs from sugarbeet bagasse using laser ablation, and we characterized the silica NPs using environmental scanning electron microscopy (ESEM), energy dispersive spectrometry (EDS), dynamic light scattering (DLS), transmission electron microscopy (TEM), attenuated total reflectanceFourier transform infrared spectroscopy (ATR-FTIR), X-ray photoelectron spectroscopy (XPS) and Raman spectroscopy. EDS analysis and XPS confirmed the presence of silica NPs. The NPs produced by laser ablation were smaller $(38-190 \mathrm{~nm})$ than those produced by $\mathrm{NaOH}$ treatment $(531-825 \mathrm{~nm})$. Finally, we demonstrated positive effects of silica NPs produced from laser ablation on the growth of microalgae, and thus, our novel method may be beneficial as an environmentally friendly procedure to produce NPs.

(c) 2014 Published by Elsevier B.V. on behalf of Chinese Society of Particuology and Institute of Process Engineering, Chinese Academy of Sciences.
\end{abstract}

\section{Introduction}

Silica is beneficial to many plants (Ding, Ma, Shui, Wan, \& Li, 2005). It is well known that certain plants, including grasses (Poaceae), rice (Oryza sativa), sugarbeet (Beta vulgaris), and horsetail (Equisetum), contain high levels of biogenic silica (Sun \& Gong, 2001). In particular, sugarbeet is an attractive source of biogenic silica because the silica content of this plant is mainly concentrated in bagasse. Sugarbeet bagasse is produced in large quantities as an agro-industrial byproduct and is often used as boiler fuel for generating steam during the processing of sugar.

\footnotetext{
* Corresponding author. Tel.: +90 312290 3526; fax: +90 3122664365 .

** Corresponding author at: Polatlı Science and Literature Faculty, Biology Department, Gazi University, Ankara 06900, Turkey. Tel.: +90 31248462 70; fax: +903124846271.

E-mail addresses: ortac@unam.bilkent.edu.tr (B. Ortaç), ttekinay@gazi.edu.tr (T. Tekinay).
}

In recent years, there has been an increasing trend toward the more efficient use of agro-industrial by-products for animal nutrition, fuel, and fermentative products. Several processes and products using sugarbeet bagasse as the raw material have been reported, particularly in pulp and paper production; it is also used as a feedstock in fermentation processes (Alves, Felipe, Silva, Silva, \& Prata, 1998; Pandey, Soccol, Nigam, \& Soccol, 2000). However, sugarbeet bagasse can also be processed to produce high-purity silica, exceeding $99 \%$ purity and primarily bearing $\mathrm{K}_{2} \mathrm{O}$, and $\mathrm{MgO}$ as impurities (Affandi, Setyawan, Winardi, Purwanto, \& Balgis, 2009). As such, bagasse is an economically viable raw material for silica nanoparticle (NP) production.

Nanoparticles are frequently used in several nanotechnological applications. In particular, silica NPs are widely used in drugs, cosmetics, printer toners, varnishes, and food preservatives (Baek \& An, 2011; Bagwe, Hilliard, \& Tan, 2006; Hua et al., 2009; Lin, Huang, Zhou, \& Ma, 2006). In addition, the use of silica NPs has recently been extended to the biomedical and biotechnological 
fields (Clément et al., 2013; Trewyn, Giri, Slowing, \& Lin, 2007). Such trends necessitate the development of eco-friendly processes for the production of NPs. As a result, the focus of NP synthesis has shifted away from physical and chemical processes toward 'green' chemistry and bioprocesses (Gonzalez-Arellano, Balu, Luque, \& Macquarrie, 2010; Tolaymat et al., 2010; Zhao \& Zhu, 2009).

To date, many approaches have been developed for silica NP synthesis. Generally, silica precursors, such as silicon alkoxides, are used as the silicon source, and NPs are generated by hydrolysis and subsequent polycondensation (Cadby \& Tolbert, 2005; Molenkamp, Watanabe, Miyata, \& Tolbert, 2004; Suzuki, Kiba, \& Yamauchi, 2011). Such chemical processes are energy intensive and thus expensive. In addition, they are usually associated with high temperatures, strong acidities, and high pressures that render NP synthesis ecologically hazardous (Bansal, Ahmad, \& Sastry, 2006). Considering the increasing demand for silica NPs in new applications (Lee, Park, Singha, \& Kim, 2013), current approaches for silica NP synthesis may be unsustainable and cost prohibitive in the near future. Therefore, it is highly desirable to identify alternative approaches to reduce production costs.

Most NP synthesis techniques, such as physical vapor deposition (Yousefi \& Muhamad, 2010), precipitation (Yang \& Hu, 2010), solvothermal/hydrothermal methods (Wang, Shi, Qi, \& Liu, 2010), and sol-gel methods (e.g., sol-gel combustion), are expensive and complex and offer only limited control over particle size and size uniformity. In recent years, pulsed-laser ablation of solids in solution has attracted interest due to its versatility and low cost (Alkis, Oruç, Ortaç, Koşger, \& Okyay, 2012; Amendola \& Meneghett, 2009; Wu, Dickinson, \& Lele, 2012). Sajti, Sattari, Chichkov, and Barcikowski (2010) demonstrated recently the bulk synthesis of NPs by laser ablation, yielding ceramic NPs on a scale of several grams. Their study indicates the potential feasibility of laser ablation for large-scale synthesis applications.

Based on the available literature (Affandi et al., 2009), we hypothesized that sugarbeet bagasse, which is inherently rich in silica, can be used to synthesize silica NPs. In this study, we describe for the first time the use of laser ablation for the synthesis of silica NPs from agro-industrial byproducts. It is also important to investigate the effects of nanomaterial exposure on the aquatic environment. Green algae are known to be sensitive to many chemicals. They have been considered indicators of the bioactivity of industrial wastes, and they vary in their responses to a variety of toxicants. Their ecological position at the base of most aquatic food webs and their essential roles in nutrient cycling and oxygen production are critical to many ecosystems. Therefore, we examined how silica NPs impact the growth of a freshwater green algae species that is among the most widespread of all algae: Chlorella vulgaris. Our results may aid the development of environmentally friendly and economically attractive alternatives to current NP production methods.

\section{Materials and methods}

\subsection{Nanoparticle production}

Sugarbeet bagasse was obtained from the Ankara Sugar Factory, Etimesgut, Ankara, Turkey. Two separate treatments were adopted to extract silica from the bagasse samples. In the first approach, bagasse ash was obtained by calcining sugarbeet bagasse at $500^{\circ} \mathrm{C}$ for $12 \mathrm{~h}$. One gram of bagasse ash was then treated with concentrated $\mathrm{HCl}: \mathrm{HNO}_{3}=1: 3(\mathrm{v} / \mathrm{v})$ at $35^{\circ} \mathrm{C}$ for $2 \mathrm{~h}$ and oven-dried at $60^{\circ} \mathrm{C}$. Then, $50 \mathrm{~mL}$ of water was added to the residue, and the solution was alkalized to a $\mathrm{pH}$ of 13-14 with concentrated $\mathrm{NaOH}$. Following overnight incubation, the alkaline solution was neutralized with $\mathrm{HCl}$. For the second approach, the raw sugarbeet bagasse sample was used directly as the laser ablation target, without any purification or cleaning procedure. The generation of NPs from sugarbeet bagasse was achieved with a commercial nanosecond pulsed Nd:YLF laser (Empower Q-Switched Laser, Spectra Physics) operating at $527 \mathrm{~nm}$ with a pulse duration of $100 \mathrm{~ns}$ and an average output power of $16 \mathrm{~W}$ at a pulse repetition rate of $1 \mathrm{kHz}$ corresponding to a pulse energy of $16 \mathrm{~mJ}$. Raw bagasse was placed in a glass vessel containing $25 \mathrm{~mL}$ of pure deionized water. The laser beam was focused on the target using a plano-convex lens with a focal length of $50 \mathrm{~mm}$; the depth of the liquid layer above the target was approximately $5 \mathrm{~mm}$. Laser ablation was carried out for $\sim 5$ min by scanning the laser beam over the target surface. The formation of NPs in water was observed visually during laser ablation, as samples had changed in color to light yellow by the end of the irradiation. In addition to producing silica NPs, laser irradiation damaged the fibrous structure of the sugarbeet bagasse. Therefore, samples were filtered with a $0.22 \mu \mathrm{m}$ filter to remove the fibers from the NP solution.

\subsection{Nanoparticle characterization}

The morphology and elemental composition of raw bagasse was measured by an environmental scanning electron microscope with EDS (ESEM, Quanta 200 FEG, FEI Instruments, USA). The particle size and distribution of particles dispersed in distilled water were measured using dynamic light scattering (DLS) (Malvern Instruments Ltd., Malvern, UK). The stability of the silica NPs was measured from the zeta potential of the solution (NanoZS, Malvern Instruments Ltd., Malvern, UK). The morphology of silica NPs was also analyzed using a FEI Tecnai G2 F30 transmission electron microscope (TEM) connected to a high resolution imaging system. NP samples were prepared by drying a total of $2 \mu \mathrm{L}$ of the laser ablated mixture on carbon coated copper grids at room temperature.

Fourier transform infrared spectroscopy (ATR/FTIR) analysis was performed using a Nicolet 6700 (Thermo Fisher Scientific, USA) ATR-FTIR spectrometer. Spectra were obtained within the $4000-500 \mathrm{~cm}^{-1}$ range with a resolution of $4 \mathrm{~cm}^{-1}$ (Bruker, Vertex 70 with Hyperion Scanning Microscope, Germany). The samples $(100 \mu \mathrm{L})$ were placed in the attenuated total reflectance (ATR, ZnSe) analyzer and analyzed.

X-ray photoelectron spectroscopy (XPS) (K $\alpha$-Monochromated high performance) (Thermo, USA) measurements were performed in an ultra-high vacuum (UHV) with a conventional X-ray source $(\mathrm{Mg}-\mathrm{K} \alpha)$.

Raman spectra were acquired at room temperature by using a Witec Alpha300S + Raman Module (Witec, Germany). A solid-state $532 \mathrm{~nm}$ wavelength laser was used for excitation. Raman measurements of single spectra were taken at $50 \times$ magnification and with $2.03 \mathrm{~s}$ integration times.

\subsection{Effects of silica nanoparticles on C. vulgaris growth}

The alga C. vulgaris was obtained from a culture collection at Gazi University Life Sciences Application and Research Center and sub-cultured in the laboratory. C. vulgaris was cultivated in sterilized Tap medium (Tris-acetate-phosphate) under an illumination intensity of 4000 lux. The temperature in the air-conditioned growth chamber was maintained at $25^{\circ} \mathrm{C}$. We exposed silica NPs produced from two different treatments to algal cultures. The algal density of three replicates was then calculated by measuring the optical density (OD) at a wavelength of $750 \mathrm{~nm}$ with a UV-vis spectrophotometer (Shimadzu, UV-1201V, Japan). Medium without NPs inoculated with alga was used as the control. Every day, 


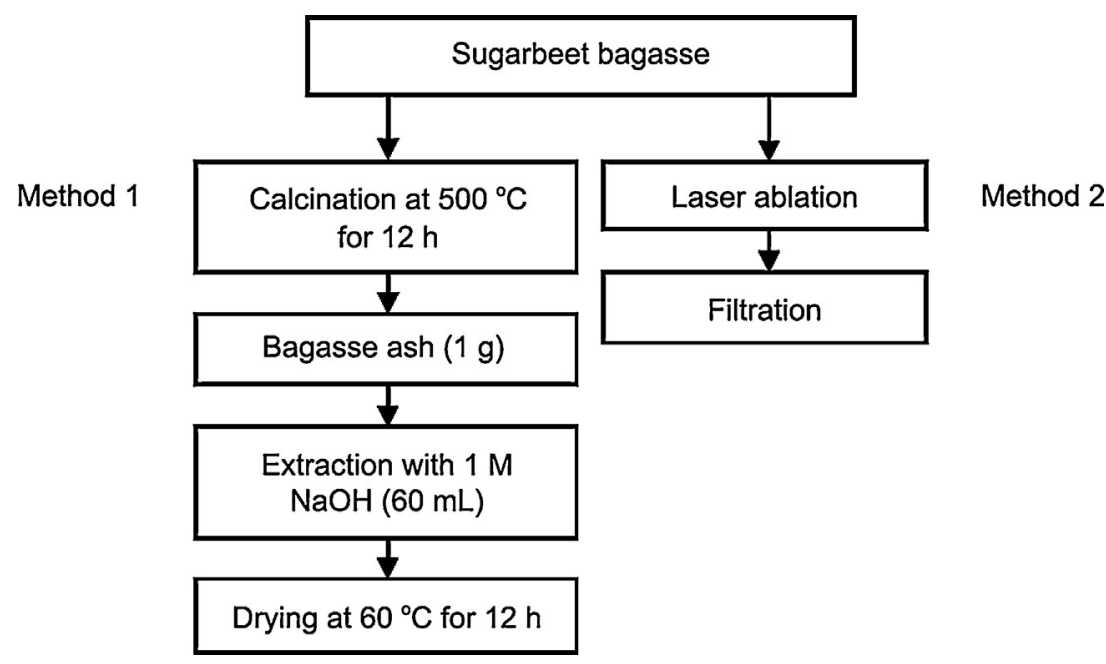

Fig. 1. Flow diagram of the procedure used to produce silica nanoparticles from sugarbeet bagasse.
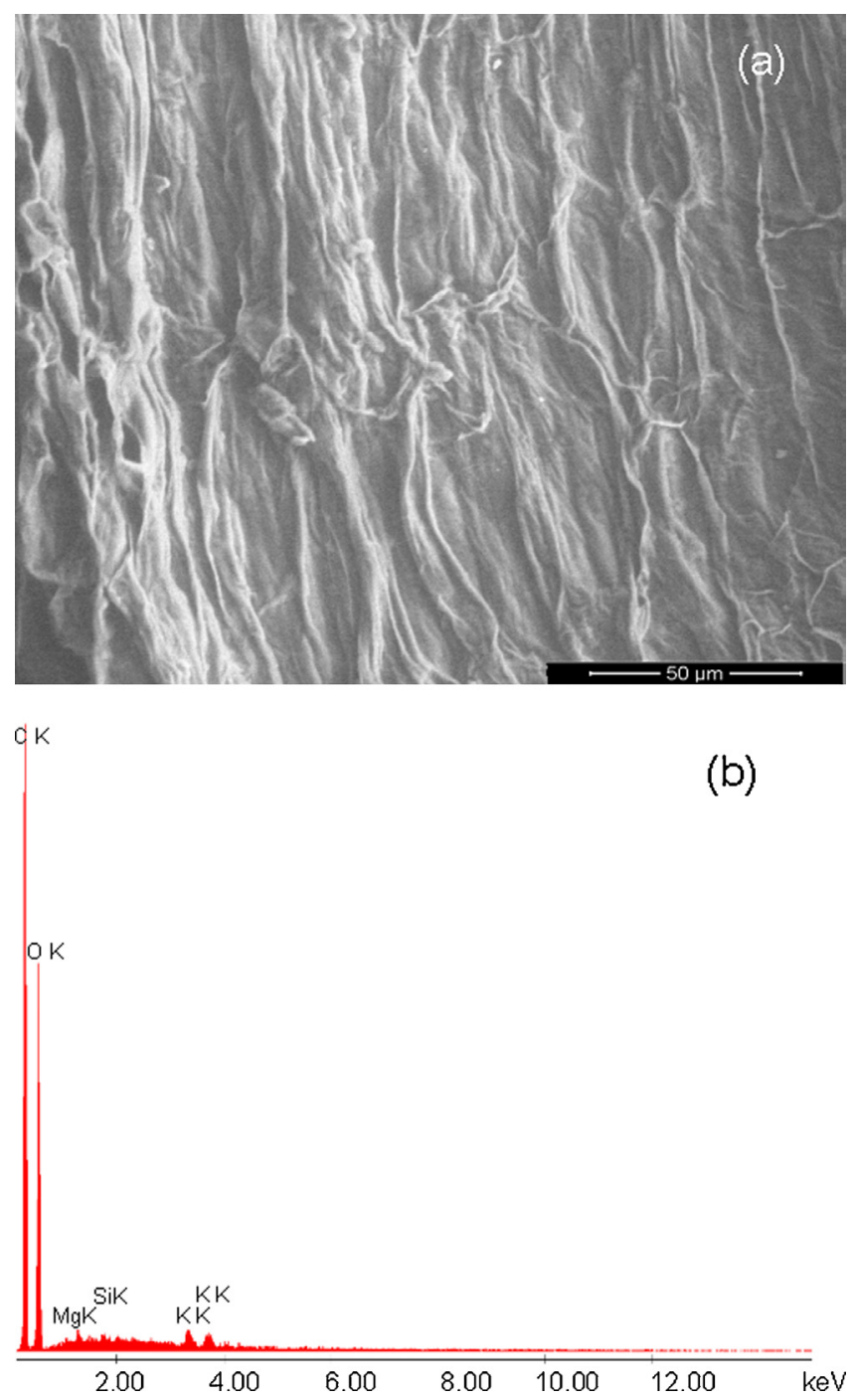

Fig. 2. (a) ESEM images and (b) EDS spectrum of raw sugarbeet bagasse. cultures were visually inspected for contamination using a light microscope.

\section{Results and discussion}

In the present study, the silica NPs were prepared using two different methods: (1) calcination of sugarbeet bagasse and subsequent treatment of sugarbeet bagasse ash with $\mathrm{NaOH}$ and (2) synthesis of sugarbeet bagasse using laser ablation (Fig. 1).

Fig. 2(a) and (b) shows the ESEM images and EDS spectrum, respectively, of raw bagasse. The ESEM micrograph of raw sugarbeet bagasse (Fig. 2(a)) clearly reveals its fibrous texture. In
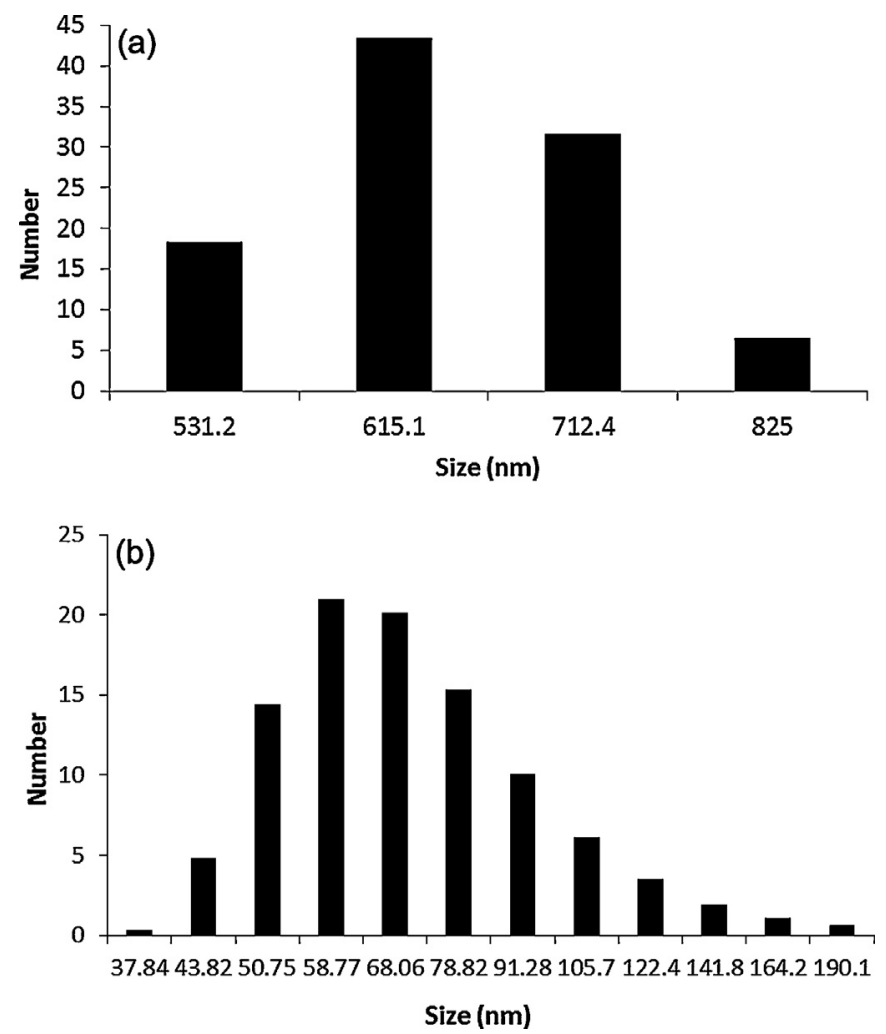

Fig. 3. The particle number distributions of silica NPs obtained by (a) $\mathrm{NaOH}$ treatment and (b) laser ablation. 

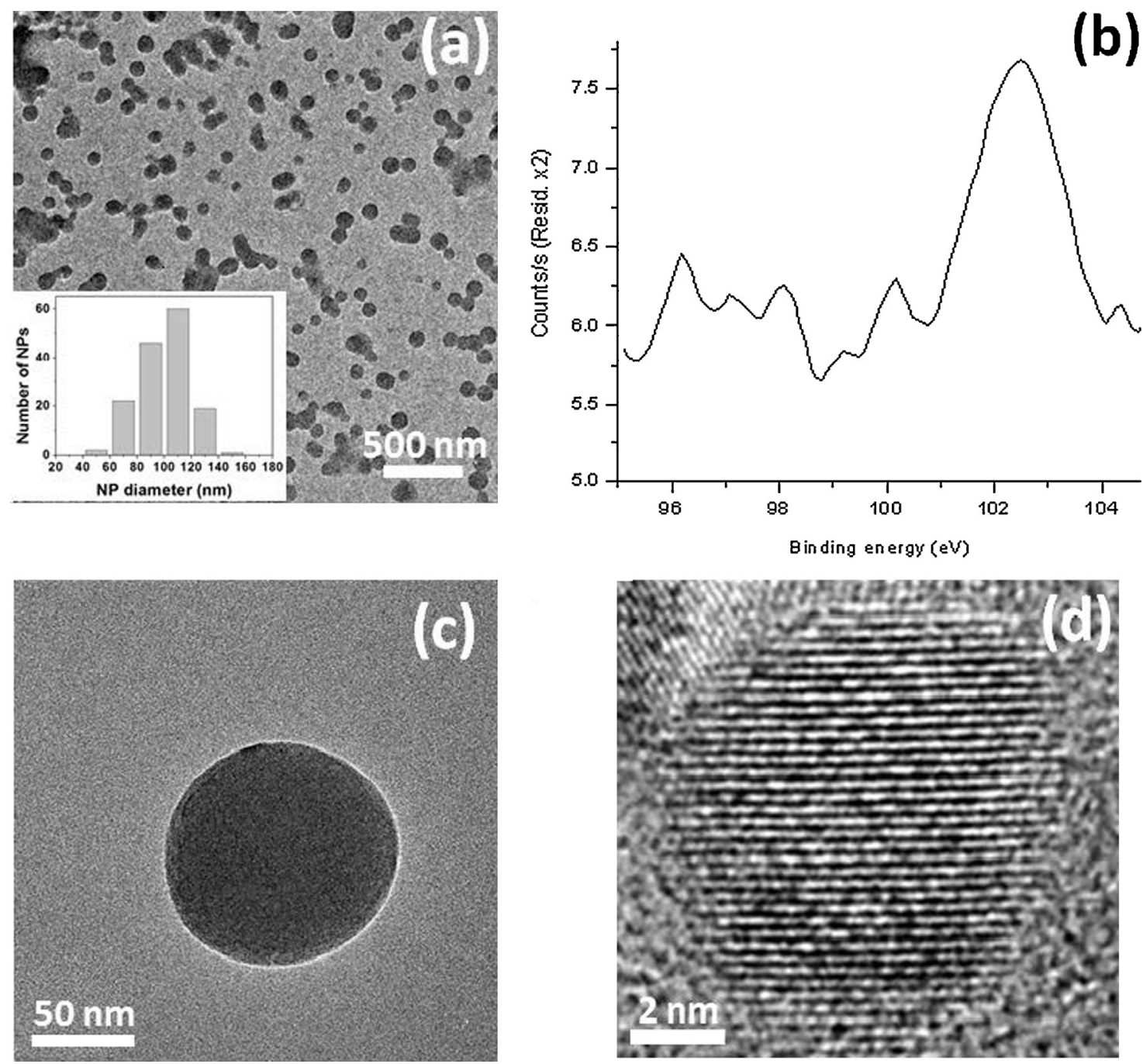

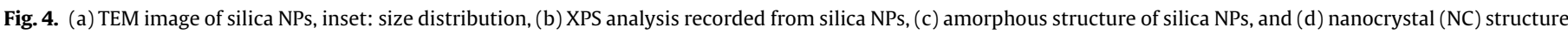
of silica NPs.

addition, EDS analysis showed that raw sugarbeet bagasse was primarily composed of metal oxides, such as Mg, K, and Si (Fig. 2(b)).

Fig. 3 shows the intensity and particle number distributions of silica NPs of various sizes after (a) $\mathrm{NaOH}$ treatment and (b) laser ablation. Fig. 3(a) demonstrates that the silica particles produced by calcination and $\mathrm{NaOH}$ treatment were substantially larger than those formed via laser ablation, with a size range of 531-825 nm. In contrast, silica NPs formed via laser ablation were in the range of 38-190 nm, with an average size of $\sim 74 \mathrm{~nm}$ (Fig. 3(b)).

A detailed TEM analysis was performed to identify the structural properties of the colloidal nanoparticle solution (CNS). Fig. 4 shows that the CNS was successfully produced by pulsed laser ablation method in deionized water. The CNS consists of spheroid-shaped and uniformly dispersed nanoparticles, without any aggregation. In addition, zeta potential measurements were carried out, and the zeta potential was $-21.0 \mathrm{mV}$, indicating that the silica NPs were stable. The data indicate the CNS in deionized water was stable and well dispersed after the laser ablation process. To obtain accurate particle size information, we measured the sizes of 150 particles observed in the TEM images. As apparent from the inset of Fig. 4(a), nanoparticles ranged in size between 40 and $160 \mathrm{~nm}$, with an average particle size of $100 \mathrm{~nm}$. To verify the chemical composition of the CNS, XPS analysis was performed. The peak at $102.43 \mathrm{eV}$ corresponds to the Si 2p spectra, indicating the existence of $\mathrm{Si}-\mathrm{O}$ bonds of $\mathrm{SiO}_{2}$ nanoparticles (Yang, Kuperman, Coombs, MamicheAfara, \& Ozin, 1996). These data show that the colloidal NP solution consists of $\mathrm{SiO}_{2}$ NPs (Fig. 4(b)). In addition, Fig. 4(a) shows that the one-step production method yielded NPs with amorphous and crystal structure. Fig. 4(c) shows an isolated amorphous NP, and Fig. 4(d) shows an isolated nanocrystal (NC). The lattice-fringe structure apparent in the HR-TEM image of the single isolated NC evidences the generation of nanoparticles in the form of a crystalline structure by laser ablation technique.

Production of Si NPs has previously been reported from silicacontaining agro-industrial waste, such as rice husk ash and coffee (Estevez, Vargas, Castaño, \& Rodriguez, 2009; Li \& Wang, 2008). In these studies, Si NPs were obtained by ashing followed by a chemical treatment of $6 \mathrm{~h}$ or longer. Our results demonstrate that laser ablation can be used as a one-step, uncomplicated method to produce silica NPs that are significantly smaller than those produced by chemical methods, such as calcination followed by $\mathrm{NaOH}$ treatment.

Infrared spectra of the silica NPs synthesized using two different methods were recorded by FTIR spectroscopy and are presented in Fig. 5. As seen in Fig. 5, silica peaks are clearly visible. The predominant absorbance peaks of silica are displayed in the low-frequency 


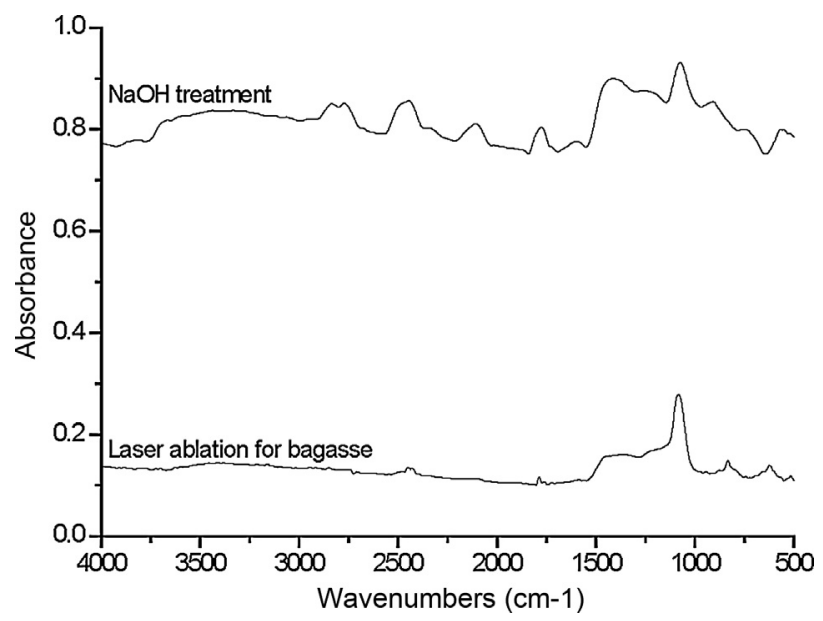

Fig. 5. FTIR spectra of NPs obtained from laser ablation of bagasse and $\mathrm{NaOH}$-treated bagasse ash.

region. The bands at $1200-1000 \mathrm{~cm}^{-1}$ and 807 are due to the asymmetric and symmetric stretching modes of $\mathrm{Si}-\mathrm{O}-\mathrm{Si}$ (Beganskiene, Sirutkaitis, Kurtinaitiené, Juškėnas, \& Kareiva, 2004). The band at $955 \mathrm{~cm}^{-1}$ was ascribed to the SiO-H asymmetry bending vibration. The broad peak at approximately $3200-3600 \mathrm{~cm}^{-1}$ corresponds to the stretching vibrations of hydroxyl groups, whereas the band at $1630-1640 \mathrm{~cm}^{-1}$ is due to the deformation of water molecules absorbed through the silica particle surface (Martinez, Ruiz, Vorobiev, Perez-Robles, \& Gonzalez-Hernandez, 1998). The peaks centered at approximately $1100 \mathrm{~cm}^{-1}$ show an obvious broadening and a shoulder in the $1160-1290 \mathrm{~cm}^{-1}$ range. This pattern can be attributed to the asymmetric stretching vibrations of the tetrahedral $\mathrm{SiO}_{4}$ coordination units (Pol, Gedanken, \& CalderonMoreno, 2003; Wang et al., 2011). Similarities between the spectra of the two silica samples indicate that the pretreatment does not affect the chemical structure of the synthesized silica, although $\mathrm{NaOH}$ treatment produced more residues than the laser ablation method.

Through its sensitivity to vibrational properties, Raman scattering offers a valuable tool for understanding structural aspects of materials (Dreschera \& Kneipp, 2012; Woods \& Bain, 2012). In this work, the Witec Alpha 300S + Raman Module with $532 \mathrm{~nm}$ excitation was used to characterize the bond properties of silica NPs. The results of Raman scattering in the spectral range of $300-3600 \mathrm{~cm}^{-1}$ are shown in Fig. 6. Several differences are apparent among the Raman spectra of the three samples. The noise level in the $\mathrm{NaOH}$ treatment spectra is much higher than that of the laser ablation treatment, although both spectra were recorded under the same conditions. The probable source of this high noise is the larger amount of pore water incorporated in NPs due to $\mathrm{NaOH}$ treatment. We found a notable peak at $522 \mathrm{~cm}^{-1}$ in both treatments, which is close to the value observed for bulk silicon (Inada, Nakagawana, Umezu, \& Sugimura, 2002).

Fig. 7 shows the optical density (OD) of C. vulgaris exposed to silica NPs produced by laser ablation and $\mathrm{NaOH}$ treatment. Understanding the effects of nanomaterial exposure on the aquatic environment is extremely important. Because of their widespread use, NPs will likely move into aquatic, terrestrial, and atmospheric environments. Therefore, concerns have been raised about the potential environmental risks posed by NPs. Green algae are known to be sensitive to many chemicals, and they are considered indicators of the bioactivity of industrial wastes. Their ecological position at the base of most aquatic food webs and their essential roles in
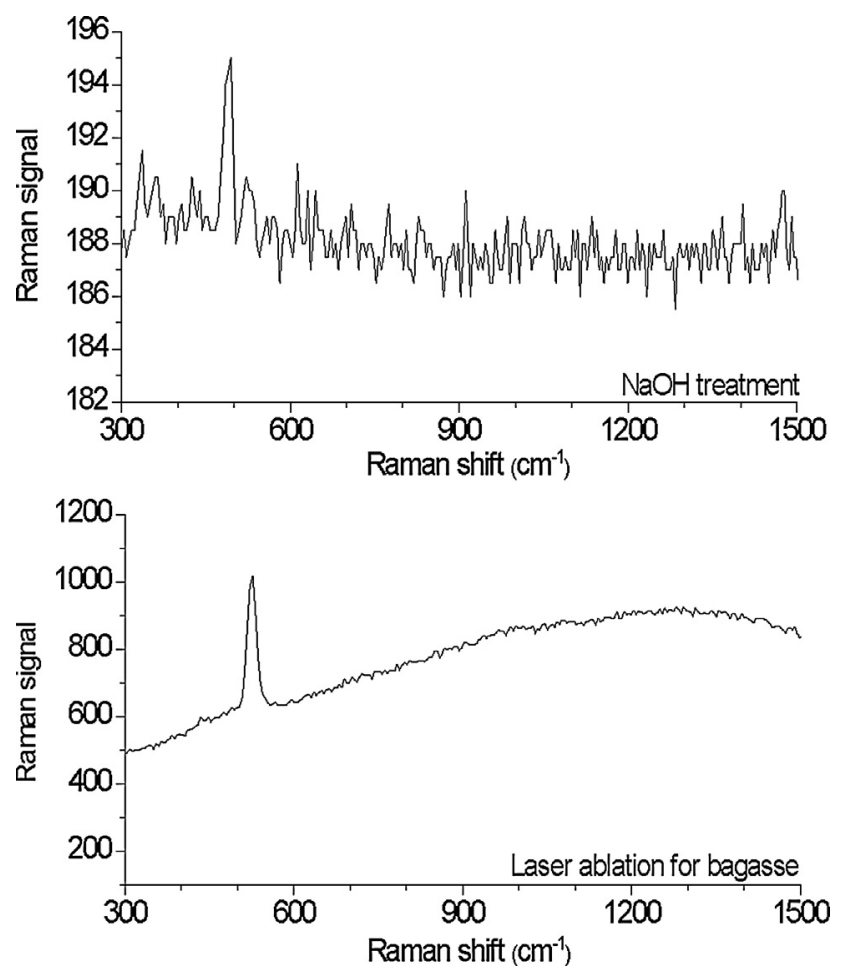

Fig. 6. Raman scattering spectra of NPs resulting from laser ablation of bagasse and $\mathrm{NaOH}$ treated bagasse ash.

nutrient cycling and oxygen production are critical to many ecosystems. However, few studies have investigated NP toxicity to algae.

Zhu, Zhu, Tian, Lang, and Li (2008) studied the toxicity of ZnO, $\mathrm{CuO}$, and $\mathrm{TiO}_{2}$ NPs to the green algae Scenedesmus obliquus. They found that ZnO NPs were the most toxic, followed by CuO NPs and $\mathrm{TiO}_{2}$ NPs. In the present study, Si NPs did not inhibit algal growth but instead increased the growth rate.

Wei et al. (2010) showed that $\mathrm{SiO}_{2}$ NPs of $10-20 \mathrm{~nm}$ in diameter were toxic to S. obliquus. They found that the smaller the particle, the greater its toxicity. The toxicity of Si NPs is most likely due to their sorption to the algal cell surface. In the present study, the size of NPs produced by laser ablation was $38-190 \mathrm{~nm}$. Therefore, we speculate that the laser-ablated Si NPs cannot sorb to the cell surface. In addition, the morphology of the algal cells did not change when observed under an optical microscope.

Major nutrients, including carbon, nitrogen, phosphorus, and silica, are defined as such because they are essential for life in

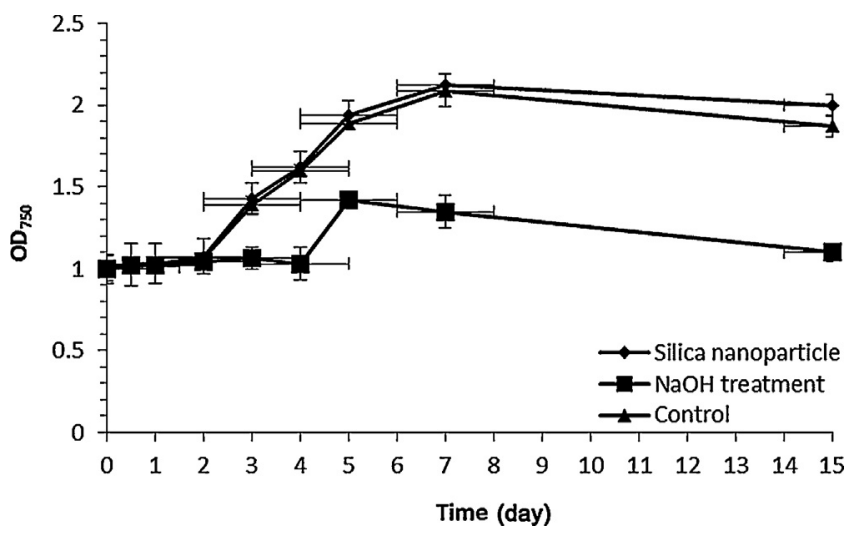

Fig. 7. Effects of silica NPs produced from laser ablation and $\mathrm{NaOH}$ treatment on growth of Chlorella vulgaris. 
aquatic organisms. Other potentially important nutrients include calcium, magnesium, sodium, potassium, and sulfur. Nutrients are important for water quality for several reasons, but often they are associated with algal growth. Our laser ablated solution contains these nutrients; therefore, we hypothesize that these nutrients may have increased the algae growth.

The $\mathrm{pH}$ range for most cultured algal species is between 7 and 9. However, in our chemical treatment step, the $\mathrm{pH}$ was first raised to $13-14$, followed by overnight incubation and neutralization of the solution with $\mathrm{HCl}$. We speculate that due to the changes in $\mathrm{pH}$, algal growth did not increase. However, more studies are needed to illustrate the mechanisms of microbial responses to NPs.

\section{Conclusions}

Silica is a key material utilized extensively in a wide range of applications. The preparation of silica NPs from sugarbeet bagasse not only takes full advantage of the high silica content of sugarbeet bagasse but also minimizes the environmental issues associated with current applications and disposal methods of sugarbeet bagasse. As such, the method presented herein may aid the development of new economical approaches involving the synthesis of valuable nanomaterials from agro-industrial residues as alternatives to the energy-intensive and potentially hazardous processes currently adopted by industry.

Here, we report the successful preparation of environmentally friendly silica NPs using laser ablation, as confirmed by SEM, TEM, and DLS. Our results show that the NPs obtained by laser ablation are significantly smaller (38-190 $\mathrm{nm}$ ) than those prepared using chemical treatment.

Last, this study demonstrates the effect of silica NPs produced using two different nanoparticle methods on algal growth. From our results, we conclude that silica NPs produced from chemical treatment have a negative effect on aquatic algae, as manifested by the decreased growth of algal cells. However, silica NPs produced by laser ablation increased the growth of $C$. vulgaris. Our results suggest that silica NPs produced by laser ablation may pose no harm to the aquatic environment.

\section{Acknowledgments}

The authors thank Zeynep Ergül Ülger for procuring the sugarbeet bagasse samples, Ömer Faruk Sarıoglu for help in analyzing the ATR/FTIR data, Alper Devrim Özkan for obtaining the Raman spectra and Ahmet Emin Topal for performing the XPS analysis.

\section{References}

Affandi, S., Setyawan, H., Winardi, S., Purwanto, A., \& Balgis, R. (2009). A facile method for production of high-purity silica xerogels from bagasse ash. Advanced Powder Technology, 20, 468-472

Alkis, S., Oruç, F. B., Ortaç, B., Koşger, A. C., \& Okyay, A. K. (2012). A plasmonic enhanced photodetector based on silicon nanocrystals obtained through laser ablation. Journal of Optics, 14, 125001.

Alves, L. A., Felipe, M. G. A., Silva, J. B. A. E., Silva, S. S., \& Prata, A. M. R. (1998). Pretreatment of sugarcane bagasse hemicellulose hydrolysate for xylitol production by Candida guilliermondii. Applied Biochemistry and Biotechnology, 70-72, 89-98.

Amendola, W. V., \& Meneghett, M. (2009). Laser ablation synthesis in solution and size manipulation of noble metal nanoparticles. Physical Chemistry Chemical Physics, 11, 3805-3821.

Baek, Y. W., \& An, Y.-J. (2011). Microbial toxicity of metal oxide nanoparticles (CuO, $\mathrm{NiO}, \mathrm{ZnO}$, and $\mathrm{Sb}_{2} \mathrm{O}_{3}$ ) to Escherichia coli, Bacillus subtilis, and Streptococcus aureus. Science of the Total Environment, 409, 1603-1608.

Bagwe, R. P., Hilliard, L. R., \& Tan, W. H. (2006). Surface modification of silica nanoparticles to reduce aggregation and non-specific binding. Langmuir, 22, 43574362.

Bansal, V., Ahmad, A., \& Sastry, M. (2006). Fungus-mediated biotransformation of amorphous silica in rice husk to nanocrystalline silica. Journal of the American Chemical Society, 128(43), 14059-14066.
Beganskienè, A., Sirutkaitis, V., Kurtinaitienė, M., Juškènas, R., \& Kareiva, A. (2004) FTIR, TEM and NMR investigations of Stöber silica nanoparticles. Journal of Materials Science, 10, 287-290.

Cadby, A. J., \& Tolbert, S. H. (2005). Controlling optical properties and interchain interactions in semiconducting polymers by encapsulation in periodic nanoporous silicas with different pore sizes. The Journal of Physical Chemistry B 109, 17879-17886.

Clément, L., Zenerino, A., Hurel, C., Amigoni, S., Taffin de Givenchy, E., Guittard, F. et al. (2013). Toxicity assessment of silica nanoparticles, functionalised silica nanoparticles, and HASE-grafted silica nanoparticles. Science of the Total Environment, 450-451, 120-128.

Ding, T. P., Ma, G. R., Shui, M. X., Wan, D. F., \& Li, R. H. (2005). Silicon isotope study on rice plants from the Zhejiang Province, China. Chemical Geology, 218(1) 41-50.

Dreschera, D., \& Kneipp, J. (2012). Nanomaterials in complex biological systems, insights from Raman spectroscopy. Chemical Society Reviews, 41, 57805799 .

Estevez, M., Vargas, S., Castaño, V. M., \& Rodriguez, R. (2009). Silica nano-particles produced by worms through a bio-digestion process of rice husk. Journal of NonCrystalline Solids, 355, 844-850.

Gonzalez-Arellano, C., Balu, A. M., Luque, R., \& Macquarrie, D. J. (2010). Catalytically active self-assembled silica-based nanostructures containing supported nanoparticles. Green Chemistry, 12, 1995-2002.

Hua, D., Tang, J., Jiang, J. L., Gu, Z. Q., Dai, L. L., \& Zhui, X. L. (2009). Controlled grafting modification of silica gel via RAFT polymerization under ultrasonic irradiation. Materials Chemistry and Physics, 114, 402-406.

Inada, M., Nakagawana, H., Umezu, I., \& Sugimura, A. (2002). Effects of hydrogen on S nanoparticles formed by pulsed laser ablation. Applied Surface Science, 197-198, 666-669.

Lee, J., Park, J., Singha, K., \& Kim, W. J. (2013). Mesoporous silica nanoparticle facilitated drug release through cascade photosensitizer activation and cleavage of singlet oxygen sensitive linker. Chemical Communication, 49, 15451547

Li, T., \& Wang, T. (2008). Preparation of silica aerogel from rice hull ash by drying at atmospheric pressure. Materials Chemistry and Physics, 112, 398401.

Lin, W., Huang, Y. W., Zhou, X. D., \& Ma, Y. (2006). In vitro toxicity of silica nanoparticles in human lung cancer cells. Toxicology and Applied Pharmacology, 217 252-259.

Martinez, J. R., Ruiz, F., Vorobiev, Y. V., Perez-Robles, F., \& Gonzalez-Hernandez J. (1998). Infrared spectroscopy analysis of the local atomic structure in silica prepared by sol-gel. The Journal of Chemical Physics, 109(17), 75117514.

Molenkamp, W. C., Watanabe, M., Miyata, H., \& Tolbert, S. H. (2004). Highly polarized luminescence from optical quality films of a semiconducting polymer aligned within oriented mesoporous silica. Journal of the American Chemical Society, 126(14), 4476-4477.

Pandey, A., Soccol, C. R., Nigam, P., \& Soccol, V. T. (2000). Biotechnology for agroindustrial residues utilisation. I: Sugarcane bagasse. Bioresource Technology, 74, 69-80.

Pol, V. G., Gedanken, A., \& Calderon-Moreno, J. (2003). Deposition of gold nanoparticles on silica spheres: A sonochemical approach. Chemistry of Materials, 15(5), 1111-1118.

Sun, L., \& Gong, K. (2001). Review: Silicon-based materials from rice husks and their applications. Industrial \& Engineering Chemistry Research, 40(25), 5861 5877.

Tolaymat, T. M., El Badawy, A. M., Genaidy, A., Scheckel, K. G., Luxton, T. P., \& Suidan, M. (2010). An evidence-based environmental perspective of manufactured silver nanoparticle in syntheses and applications: A systematic review and critica appraisal of peer-reviewed scientific papers. Science of the Total Environment, 408, 999-1006.

Trewyn, B. G., Giri, S., Slowing, I. I., \& Lin, V. S. Y. (2007). Mesoporous silica nanoparticle based controlled release, drug delivery, and biosensor systems. Chemica Communications, 31, 3236-3245.

Sajti, C. L., Sattari, R., Chichkov, B. N., \& Barcikowski, S. (2010). Gram scale synthesis of pure ceramic nanoparticles by laser ablation in liquid. The Journal of Physical Chemistry C, 114, 2421-2427.

Suzuki, N., Kiba, S., \& Yamauchi, Y. (2011). Fabrication of mesoporous silica/polyme composites through solvent evaporation process and investigation of their excellent low thermal expansion property. Physical Chemistry Chemical Physics, 13(11), 4957-4962.

Wang, J., Shi, N., Qi, Y., \& Liu, M. (2010). Reverse micelles template assisted fabrication of $\mathrm{ZnO}$ hollow nanospheres and hexagonal microtubes by a novel fast microemulsion-based hydrothermal method.Journal of Sol-Gel Science and Technology, 53, 101-106.

Wang, W., Martin, J. C., Zhang, N., Ma, C., Han, A., \& Sun, L. (2011). Harvesting silica nanoparticles from rice husks. Journal of Nanoparticle Research, 13, 69816990

Wei, C., Zhang, Y., Guo, J., Han, B., Yang, X., \& Yuan, J. (2010). Effects of silica nanoparticles on growth and photosynthetic pigment contents of Scenedesmus obliquus. Journal of Environmental Sciences, 22, 155-160.

Woods, A. D., \& Bain, C. D. (2012). Total internal reflection Raman spectroscopy. Analyst, 137, 35-48

Wu, J., Dickinson, R. B., \& Lele, T. P. (2012). Investigation of in vivo microtubule and stress fiber mechanics with laser ablation. Integrative Biology, 4, 471-479. 
Yousefi, R., \& Muhamad, M. R. (2010). Effects of gold catalysts and thermal evaporation method modifications on the growth process of $\mathrm{Zn}_{1-x} \mathrm{Mg}_{x} \mathrm{O}$ nanowires. Journal of Solid State Chemistry, 183, 1733-1739.

Yang, H., Kuperman, A., Coombs, N., Mamiche-Afara, S., \& Ozin, G. (1996). Synthesis of oriented mesoporous silica films on mica. Nature, 379, 703-705.

Yang, Q., \& Hu, W. (2010). A novel mercury-media route to synthesize ZnO hollow microspheres. Ceramics International, 36, 989-993.
Zhao, B., \& Zhu, L. (2009). Mixed polymer brush-grafted particles: A new class of environmentally responsive nanostructured materials. Macromolecules, 42, 9369-9383.

Zhu, X. S., Zhu, L., Tian, S. Y., Lang, Y. P., \& Li, Y. (2008). Aquatic ecotoxicities of nanoscale $\mathrm{TiO}_{2}, \mathrm{ZnO}$ and $\mathrm{Al}_{2} \mathrm{O}_{3}$ water suspensions. Acta Ecologica Sinica, 28(8), 3507-3516 [in Chinese]. 